PROCEEDINGS OF THE

AMERICAN MATHEMATICAL SOCIETY

Volume 136, Number 12, December 2008, Pages 4167-4173

S 0002-9939(08)09597-X

Article electronically published on June 26, 2008

\title{
SEPARATORS OF SIEGEL MODULAR FORMS OF DEGREE TWO
}

\author{
BERNHARD HEIM
}

(Communicated by Wen-Ching Winnie Li)

\begin{abstract}
We prove that cuspidal Siegel modular forms of degree two and weight $2 k$ are uniquely determined by their Fourier coefficients on small subsets of matrices of content one. This generalizes results of Breulmann, Kohnen, Katsurada, Scharlau and Walling. We give applications to the space of SaitoKurokawa lifts.
\end{abstract}

\section{Introduction AND STATEMENT OF RESUlts}

Cuspidal Siegel modular forms of degree $n$ and weight $k$ are uniquely determined by their Fourier coefficients, which are parametrized by the set $X_{n}$ of positivedefinite $n$-by- $n$ half-integral matrices. For various purposes, it is important to determine principal separators, that is, small subsets of $X_{n}$ such that any two distinct forms of $S_{k}^{n}$ have at least one different Fourier coefficient supported on the separator.

Since Hecke's time it has been known that Hecke eigenforms for $S L_{2}(\mathbb{Z})$ are separated by the set $\mathbb{P}$ of primes. That is, two eigenforms of weight $k$ with Fourier expansions with identical prime-index Fourier coefficients are identical. A set that separates Hecke eigenforms we call a separator. The strong multiplicity-one theorem [3] further refines this fact to an arbitrary level. The complement $S^{c}$ in $\mathbb{P}$ of a finite set $S$ of primes is a separator. More generally, one wants principal separators, that is, sets $S \subset \mathbb{N}$ such that if $h \in S_{k}$ with $a_{h}(s)=0$ for $s \in S$, then $h=0$. To simplify notation we drop the index in the case $n=1$. To be a Hecke eigenform is a very strong auxiliary condition for separation. For example, the set $S^{c}$ coming from the strong multiplicity-one theorem is not known to be a principal separator, although it is a separator (for eigenforms).

It is more difficult to obtain non-trivial separation results for Siegel modular forms, since eigenvalues and Fourier coefficients are not as directly related as in the case of elliptic modular forms. For example, for Siegel modular forms of degree larger than one, no multiplicity-one theorem is known. Before stating our theorem we recall what is known in the degree 2 case. For Siegel modular forms of even weight $k$ for the Siegel modular group $S p_{2}(\mathbb{Z})$ the following result was given by

Received by the editors November 8, 2007.

2000 Mathematics Subject Classification. Primary 11Fxx.

(C)2008 Bernhard Heim 
Breulmann and Kohnen [2. The set

$$
S_{\mathrm{BK}}:=\left\{l T \mid l \in \mathbb{N} \text { square-free and } T \in X_{2} \text { primitive }\right\}
$$

is a separator.

To prove this, Breulmann and Kohnen twisted by grossencharacters an identity of Andrianov between the spinor zeta function and the Koecher-Maass series. Certain functional equations and a converse theorem of Imai gave the result. This result was reproved by Katsurada [5] and Scharlau and Walling [11. Their argument, in contrast to that of Breulmann and Kohnen, is purely algebraic and is based on combinatorical properties of Hecke operators. This argument also works for congruence subgroups and higher genus. Nevertheless, the criterion of the theorem of Breulmann and Kohnen for deciding whether two Siegel Hecke eigenforms of degree 2 are the same is the sharpest to date. All these papers make essential use of the assumption that the cuspforms to be compared are Hecke eigenforms. Without this condition the separator has to be larger. In a different direction, for fixed $k$, finite sets of principal separators have been given by Poor and Yuen 7 .

Let $\omega$ be the arithmetic function $\omega(m):=\#\{p$ prime $: p \mid m\}$ and let $\lfloor x\rfloor$ be the largest integer less than or equal to $x$. Let

$$
\mathbb{k}:=\max \left\{\operatorname{dim} S_{k+2\left\lfloor\frac{k}{10}\right\rfloor-2}, \operatorname{dim} S_{k+2\left\lfloor\frac{k}{10}\right\rfloor}\right\}
$$

for $k \in \mathbb{N}$ even $(k>8)$.

Theorem 1. For a positive even integer $k$, the subset

$$
\left\{\left(\begin{array}{cc}
n & \frac{r}{2} \\
\frac{r}{2} & m
\end{array}\right) \in X_{2} \mid n, m \text { coprime and square-free, and } \omega(n), \omega(m) \leq \mathbb{k}\right\}
$$

of primitive matrices is a principal separator of $S_{k}^{2}$.

We have two remarks.

Remark 1. The theorem implies the result of Breulmann and Kohnen [2, as it is valid for all cusp forms and as the primitive $T=\left(\begin{array}{c}n \frac{r}{2} \\ \frac{r}{2} \\ m\end{array}\right) \in X_{2}$ with coprime $m$ and $n$ are a subset of $S_{\mathrm{BK}}$.

Remark 2. The result of [2] only compares Hecke eigenforms $F, G$, and in particular assumes that $A_{F}(T)=A_{G}(T)$ for all $T \in X_{2}$ with $T=\left(\begin{array}{ll}n & \frac{r}{2} \\ \frac{r}{2} & p^{t}\end{array}\right)$, where $(p, n)=1$ and $t \geq 2$. Our theorem shows that these two conditions can be dropped.

The proof of the theorem has two main ingredients. It is built on the restricted modified derivatives of the Siegel form and on a sharp result for a principal separator of elliptic forms. The first ingredient is at the moment only available for level one 4, since questions on new and old Siegel modular forms and on the number of derivatives needed to determine the form are delicate. As pointed out by the referee, the second ingredient can be given in a much more general form. Since the result is interesting in its own right, we give one version of it. More refined results can be obtained by employing results of Ono and Skinner [6], Ramakrishnan [10] and Rajan [9]. 
Let $N \in \mathbb{N}$ and $k \geq 2$. We denote by $S_{k}\left(\Gamma_{0}(N)\right)$ the space of cusp forms with respect to

$$
\Gamma_{0}(N):=\left\{\left(\begin{array}{ll}
a & b \\
c & d
\end{array}\right) \in \mathrm{SL}_{2}(\mathbb{Z}) \mid c \equiv 0 \quad(\bmod N)\right\} .
$$

We let $S_{k}\left(\Gamma_{0}(N)\right)^{\text {new }}$ be the subspace of newforms. Then we have

Theorem 2. For a positive integer $k \geq 2$ and $N \in \mathbb{N}$ the set

$$
\left\{m \in \mathbb{N} \mid m \text { square-free and } \omega(m) \leq \operatorname{dim} S_{k}\left(\Gamma_{0}(N)\right)^{\text {new }}\right\}
$$

is a principal separator of $S_{k}\left(\Gamma_{0}(N)\right)^{\text {new }}$.

In level one, of course, all cusp forms are new. In the last section we consider the subspace of Saito-Kurokawa lifts in $S_{k}^{2}$. We determine an explicit principal separator which distinguishes lifts but is small enough to fail to be a principal separator of the entire space of Siegel modular forms.

\section{Proof of the theorems}

2.1. Proof of Theorem 2. Let $g$ be a non-trivial element in $S_{k}\left(\Gamma_{0}(N)\right)^{\text {new }}$ and $\left(f_{j}\right)_{j}$ be a basis of $S_{k}\left(\Gamma_{0}(N)\right)^{\text {new }}$ of primitive newforms with Fourier coefficients $a(n)$ and $a_{j}(n)$. Up to ordering, we can assume that

$$
g=\sum_{j=1}^{d} \alpha_{j} f_{j} \quad \text { for } 1<d \leq \operatorname{dim} S_{k}\left(\Gamma_{0}(N)\right)^{\text {new }}
$$

and $d$ minimal.

For a prime $p$ with $(p, N)=1$, consider the Hecke operators $T_{p}$ on $S_{k}\left(\Gamma_{0}(N)\right)^{\text {new }}$. The $n$-th Fourier coefficient of $T_{p}(g)$ is given by

$$
a(p n)+p^{k-1} a(n / p) .
$$

We fix a prime $p_{2}$ with $\left(p_{2}, N\right)=1$ such that $a_{1}\left(p_{2}\right) \neq a_{2}\left(p_{2}\right)$; then the $n$-th Fourier coefficient of

$$
F_{2}:=T_{p_{2}}(g)-a_{2}\left(p_{2}\right) g
$$

is given by

$$
\sum_{j, j \neq 2} \alpha_{j}^{(2)} a_{j}(n), \text { where } \alpha_{j}^{(2)}=\alpha_{j}\left(a_{j}\left(p_{2}\right)-a_{2}\left(p_{2}\right)\right) .
$$

This expression is equal to

$$
a\left(p_{2} n\right)+p_{2}^{k-1} a\left(n / p_{2}\right)-a_{2}\left(p_{2}\right) a(n) .
$$

Iterate this procedure with different prime numbers $p_{j}$ (see Lemma 24 of [1] for the existence). Eventually, we obtain $F_{d^{\prime}}=\alpha_{1}^{\left(d^{\prime}\right)} f_{1}$ with $\alpha_{1}^{\left(d^{\prime}\right)} \neq 0$ and $2 \leq d^{\prime} \leq d$. Then the $n$-th Fourier coefficient of $F_{d^{\prime}}$ is equal to

$$
\sum_{d_{1}, d_{2}} x_{d_{1}, d_{2}} a\left(\frac{d_{1}}{d_{2}} n\right) \quad \text { with } x_{d_{1}, d_{2}} \in \mathbb{Q}^{x},
$$

where the sum runs over all $d_{1}, d_{2} \in \mathbb{N}$ with $d_{1}, d_{2} \mid \prod_{i=2}^{d^{\prime}} p_{i}$ and $\left(d_{1}, d_{2}\right)=1$. Let

$$
\mathcal{P}_{g}=\left\{p \text { prime } \mid a_{1}(p) \neq 0\right\} /\left\{p_{2}, \ldots, p_{d^{\prime}}\right\} .
$$


Then it follows from our construction that there exists for every $p \in \mathcal{P}_{g}$ an $h_{p} \in \mathbb{N}$ with $h_{p} \mid p_{1} \ldots p_{d^{\prime}}$ such that $a\left(h_{p} \cdot p\right) \neq 0$. Let $\omega(m):=\#\{p$ prime $: p \mid m\}$. We have shown that the set

$$
\mathcal{N}_{k, N}:=\left\{m \in \mathbb{N} \text { square-free } \mid \omega(m) \leq \operatorname{dim} S_{k}\left(\Gamma_{0}(N)\right)^{\text {new }}\right\}
$$

is a principal separator for $S_{k}\left(\Gamma_{0}(N)\right)^{\text {new }}$.

2.2. Proof of Theorem 1. We denote by $S_{k}^{n}$ the space of Siegel cusp forms of weight $k$ and degree $n$ with respect to $\Gamma_{n}:=S p_{n}(\mathbb{Z})$ and use the special notation

$$
X_{2}=\left\{T=\left(\begin{array}{cc}
n & \frac{r}{2} \\
\frac{r}{2} & m
\end{array}\right) \mid n, m, r \in \mathbb{Z} \text { and } n, m>0 \text { with } r^{2}<4 n m\right\}
$$

in the case of degree 2. As usual we also use the parametrization $(n, r, m)$ for $T$. The content of $T$ is the greatest common divisor of $n, r$ and $m$. When the content of $T$ is one, $T$ is primitive. For the convenience of the reader, we recall some defintions and notation.

Definition. Let $\mathcal{N} \subset X_{n}$. The set $\mathcal{N}$ is a separator for a subspace $V$ of $S_{k}^{n}$ when, for Hecke eigenfunctions $F, G \in V$, if $A_{F}(T)=A_{G}(T)$ for all $T \in \mathcal{N}$, then $F=G$. We say $\mathcal{N}$ is a principal separator if the same conclusion holds without assuming that $F, G \in V$ are Hecke eigenfunctions. Moreover let $F \mid \mathcal{N}$ denote the subseries given by the Fourier coefficients indexed by $\mathcal{N}$.

Recall a result of [4]: Let $p_{k, \underline{2 \nu}}$ be the ultraspherical polynomials, i.e.,

$$
p_{k, \underline{\nu} \nu}(a, b):=\sum_{\mu=0}^{\nu}(-1)^{\mu} \frac{(2 \nu) !}{\mu !(2 \nu-2 \mu) !} \frac{(k+2 \nu-\mu-2) !}{(k+\nu-2) !} a^{2 \nu-2 \mu} b^{\mu},
$$

for $\nu, k, a, b \in \mathbb{N}_{0}$. We put

$$
A_{F}^{\nu}(n, m):=\sum_{r \in \mathbb{Z}, r<\sqrt{4 n m}} p_{k, \underline{2 \nu}}(r, n m) A_{F}(n, r, m) .
$$

Then the derivative

$$
\left(\mathcal{D}_{2 \nu} F\right)(\tau, \tilde{\tau}):=\sum_{n, m \in \mathbb{N}} A_{F}^{\nu}(n, m) e^{2 \pi i(n \tau+m \tilde{\tau})}
$$

is an element of $\operatorname{Sym}^{2} S_{k}$. For $F \neq 0$, there is a $\nu$ with $0 \leq \nu \leq\left\lfloor\frac{k}{10}\right\rfloor$ such that $\mathcal{D}_{2 \nu} F \neq 0$. This property makes it possible to obtain the claim of the theorem via properties of elliptic cusp forms.

Let $n_{0} \in \mathcal{N}_{k}:=\mathcal{N}_{k, 1}$. Then $\mathcal{N}_{k}\left(n_{0}\right):=\left\{m \in \mathcal{N}_{k} \mid\left(n_{0}, m\right)=1\right\}$ is a principal separator for $S_{k}$. This follows from the construction of $\mathcal{N}_{k}$ and the well-known fact that for a primitive Hecke eigenform $f \in S_{k}$ there exist infinitely primes $p$ such that $a_{f}(p) \neq 0$.

Let

$$
\overline{\mathcal{N}_{k}}:=\left\{(n, m) \in \mathcal{N}_{k} \times \mathcal{N}_{k} \mid(n, m)=1\right\} .
$$

We show that this is a principal separator of $\operatorname{Sym}^{2} S_{k}$. Let $\left(f_{j}\right)_{j}$ be as above and $F \in \operatorname{Sym}^{2} S_{k}$. Assume that $F \mid \overline{\mathcal{N}_{k}}=0$, and show that $F=0$. Let

$$
F(\tau, \tilde{\tau})=\sum_{i, j=1}^{\operatorname{dim} S_{k}} \alpha_{i, j} f_{i}(\tau) f_{j}(\tilde{\tau}) .
$$


For any $n \in \mathcal{N}_{k}$, for every $j$,

$$
\left(\sum_{i} \alpha_{i, j} a_{i}(n)\right) f_{j}(\tilde{\tau})=0,
$$

since $\mathcal{N}_{k}(n)$ is a principal separator of $S_{k}$. Apply the same argument again to obtain $\alpha_{i, j}=0$ for $1 \leq i, j \leq \operatorname{dim} S_{k}$. Let

$$
\mathbb{k}:=\max \left\{\operatorname{dim} S_{k+2\left\lfloor\frac{k}{10}\right\rfloor-2}, \operatorname{dim} S_{k+2\left\lfloor\frac{k}{10}\right\rfloor}\right\} .
$$

Since $\operatorname{dim} S_{2}^{2}=\operatorname{dim} S_{4}^{2}=\operatorname{dim} S_{6}^{2}=\operatorname{dim} S_{8}^{2}=0$ we can assume $k>8$. Hence, the set

$$
\{(n, m) \in \mathbb{N} \times \mathbb{N} \mid n, m \text { coprime and } \omega(n), \omega(m) \leq \mathbb{k}\}
$$

is a principal separator of

$$
\bigoplus_{\nu=0}^{\left\lfloor\frac{k}{10}\right\rfloor} \operatorname{Sym}^{2}\left(S_{k+2 \nu}\right)
$$

This proves the claim of the theorem.

\section{SAITO-KUROKAWA LIFTS}

Now consider the subspace $S K_{k}$ of Saito-Kurokawa lifts of cuspidal Siegel modular forms of degree 2, also called the Maass Spezialschar [13. We determine an explicit principal separator which distinguishes lifts but is too small to be a principal separator of the entire space of Siegel modular forms.

For a positive even integer $k$, the space $S K_{k}$ can be defined in the following way via properties of Fourier coefficients. There are other equivalent definitions if we have an eigenform with respect to the underlying Hecke algebra. Since we drop the Hecke eigenfunction assumption, we prefer the following definition.

Definition. For an even positive integer $k$,

$$
\begin{aligned}
S K_{k}=\left\{F \in S_{k}^{2} \mid A_{F}(n, r, m)=\sum_{d \mid(n, r, m)} d^{k-1} A_{F}\left(\frac{n m}{d^{2}}, \frac{r}{d}, 1\right)\right. \\
\left.\qquad \text { for all } T=(n, r, m) \in X_{2}\right\} .
\end{aligned}
$$

We can assume that the weight $k$ is even, since otherwise $S K_{k}$ is trivial.

Theorem 3. For a positive even integer $k$, the set

$$
\left\{(n, r, 1) \in X_{2} \mid n \text { square-free, and } \omega(n) \leq\left\lfloor\frac{k+2}{12}\right\rfloor\right\}
$$

is a principal separator of the space $S K_{k}$ of Saito-Kurokawa lifts.

This principal separator is optimal in the sense that it separates Saito-Kurokawa lifts, but it is too small to separate the entire space of Siegel modular forms of degree 2.

Proof. Let $F \in S K_{k}$. Then $F=0$ if and only if $\mathcal{D}_{0} F=\mathcal{D}_{2} F=0$. Moreover,

$$
\mathcal{D}_{2 \nu} F=\sum_{i=1}^{\operatorname{dim} S_{k+2 \nu}} \alpha_{i}^{\nu} f_{i}^{\nu} \otimes f_{i}^{\nu} \quad(\nu=1,2) .
$$


See 4 for details. Here $\left(\alpha_{i}^{\nu}\right)_{i}$ are complex numbers and $\left(f_{i}^{\nu}\right)_{i}$ is a basis of $S_{k+2 \nu}$ of primitive newforms. It is convenient to view $\left(\alpha_{i}^{\nu} f_{i}^{\nu}(\tau)\right)$ as a coefficient of $f_{i}^{\nu}$. This makes it possible to apply separators for $S_{k}$ and $S_{k+2}$ on

$$
h_{\tau_{0}}^{\nu}(\tilde{\tau}):=\sum_{i=1}^{\operatorname{dim} S_{k+2 \nu}}\left(\alpha_{i}^{\nu} f_{i}^{\nu}\left(\tau_{0}\right)\right) f_{i}^{\nu}(\tilde{\tau}) \in S_{k+2 \nu}
$$

for all $\tau_{0} \in \mathfrak{H}$. Since $\mathcal{N}_{k+2 \nu}$ is a principal separator of $S_{k+2 \nu}$, if all Fourier coefficients of $h_{\tau_{0}}^{\nu}$ are zero, then the function itself is zero. This immediately yields $\alpha_{i}^{\nu}=0$ for $\nu=1,2$ and $1 \leq i \leq \operatorname{dim} S_{k+2 \nu}$.

The techniques used in the last section lead to

Proposition 3.1. For a positive even integer $k$, there exist infinitely many primes $p$ such that

$$
\left\{(n, r, p) \in X_{2} \mid(n, p)=1, n \text { square-free, and } \omega(n) \leq\left\lfloor\frac{k+2}{12}\right\rfloor\right\}
$$

is a principal separator of the space $S K_{k}$ of Saito-Kurokawa lifts.

Finally, we have a special case:

Corollary 3.2. For $k=10,12,14,16,18$, the space of Siegel modular forms of degree 2 and weight $k$ has for infinitely many primes $p$ the principal separator

$$
\{(1, r, p) \mid r \in \mathbb{Z} \text { and } r<\sqrt{4 p}\} \cup\left\{(q, r, p) \in X_{2} \mid q \text { prime }(q, p)=1\right\} .
$$

\section{ACKNOWLEDGEMENT}

The author thanks the referee for valuable suggestions and a careful reading of the manuscript.

\section{REFERENCES}

[1] A. Atkin, J. Lehner: Hecke operators on $\Gamma_{0}(m)$. Math. Ann. 185 (1970), 134-160. MR0268123 (42:3022)

[2] S. Breulmann, W. Kohnen: Twisted Maass-Koecher series and spinor zeta functions. Nagoya Math. J. 155 (1999), 153-160. MR1711371 (2000g:11033)

[3] W. Casselman: On some results of Atkin and Lehner. Math. Ann. 201 (1973), 301-314. MR0337789 (49:2558)

[4] B. Heim: On the Spezialschar of Maass. arXiv: 0801.1804v1 [math.NT].

[5] H. Katsurada: On the coincidence of Hecke-eigenforms. Abh. Math. Sem. Univ. Hamburg, 70 (2000), 77-83. MR:1809535 (2001k:11083)

[6] K. Ono, C. Skinner: Fourier coefficients of half-integral weight modular forms modulo $l$. Annals of Math. (2) 147 (1998), 453-470. MR.1626761 (99f:11059a)

[7] C. Poor, D. Yuen: Linear dependence among Siegel modular forms. Math. Ann. 318 (2000), 205-234. MR $1795560(2001 \mathrm{j}: 11024)$

[8] C.S. Rajan: Refinement of strong multiplicity one for automorphic representations of $\operatorname{Gl}(n)$. Proc. Amer. Math. Soc. 128 (2000), 691-700. MR:1707005 (2000g:11041)

[9] C.S. Rajan: On strong multiplicity one for l-adic representations. IMRN 3 (1998), 161-172. MR.1606395 (99c:11064)

[10] D. Ramakrishnan: A refinement of the strong multiplicity one theorem for GL(2). Invent. Math. 116 (1994), 645-649. MR 1253208 (95h:11050b) 
[11] R. Scharlau, L. Walling: A weak multiplicity-one theorem for Siegel modular forms. Pacific J. Math. 211 (2003), 369-374. MR2015741 (2004k:11069)

[12] G. Shimura: Introduction to the Arithmetic Theory of Automorphic Functions. Iwanami Shoten, Tokyo, and Princeton Univ. Press, 1971. MR0314766 (47:3318)

[13] D. Zagier: Sur la conjecture de Saito-Kurokawa (d'après H. Maass). Sém. Delange-PisotPoitou 1979/1980, Progress in Math. 12, Birkhäuser, Boston, 1981, 371-394. MR633910 (83b:10031)

Max-Planck Institut für Mathematik, Vivatsgasse 7, 53111 Bonn, Germany

E-mail address: heim@mpim-bonn.mpg.de 